Restlessness, and other obvious symptoms of approaching labour, now supervened. The patient passed a bad night, and in the morning complained of urgent desire for, and great difficulty in, passing urine. On vaginal examination, a tumour, the size of a hen's egg, was felt in the neighbourhood of the neck of the bladder. This tumour was very hard, and perfectly immovable. As there was some history of a former injury to the pelvis, it was imagined that an exostosis might exist, but this opinion was abandoned on finding that the tumour had only occupied its present position since the rupture of the membranes-a point particularly determined. The foetal head presented immediately above and behind the tumour. On attempting to introduce the catheter, the course of the urethra seemed altered. The instrument apparently struck on some hard substance, but could not be passed into the bladder. As this organ had acted a short time before, it was deemed advisable to avoid for a time further interference. During the course of the afternoon, Dr. Cockle examined the abdomen at intervals, and was quite persuaded that the sounds of the foetal heart had definitely ceased. It was therefore suggested, should further difficulties arise, that the head should be lessened without delay.

About midnight $\mathrm{Mr}$. Hill was summoned, and finding the anguish extreme, the pressure of the head vainly attempting to advance upon the tumour, which had become more prominent than ever; the bladder distended, and the general symptoms most threatening, at once had recourse to craniotomy. The operation was most easily effected. The calculus was pushed back as carefully as possible into the bladder. the urine gushed out, and labour was terminated without further diffi. culty. It was, however, soon obvious that no attempt at rallying was likely to occur, probably from the severe mental and physical shock to which the patient had been submitted. The bladder did not recover its power of retention, and the motions passed involuntarily. In about forty-eight hours great collapse was evident. Vomiting commenced, and the abdomen began to meteorize, but without any marked pain; the pulse numbered 125; the breathing was quick and hurried; lochia highly offensive. These symptoms gradually increasing, death occurred on the 29 th.

Autopsy.-General peritonitis existed. A large amount of sero-purulent exudation was contained in the peritoneal sac. The bladder was contracted and hypertrophied, and contained the calculus which had been encysted at its neck. The bed of the cyst was perfectly defined. A narrow strap, or band of muscular tissue, stretched across the calculus, retaining it in its bed, but which had given way, probably in pressing the calculus into the bladder to facilitate delivery. The uterus was large and congested; its lining membrane throughout was in a sloughy condition.

\section{QUEEN ADELAIDE'S DISPENSARY.}

COLIOID CANCER OF THE PERITONEUM; FATAL RESULT; AUTOPSY.

(Under the care of Mr. T. J. WoonHouse.)

C. B-, a German Jew, aged sixty-four, was seen at his home, Cambridge-heath, on the 15th of February, 1861. He was extremely emaciated, and of a most cachectic appearance. He stated that his illness began four weeks ago with pains in the abdomen, back, and legs, without fever. His bowels had been, he said, habitually costive; the motions small and lumpy. He once passed eight days without any evacuation from the bowels. From the commencement of his complaint-a month ago-he noticed that his abdomen began to swell; it was greatly distended, and universally dull on percussion; fluctuation most distinct, and readily communicated from one side to the other. There was no abdominal tenderness when he was examined, although there had been at first. He thought the abdomen had become larger during the last fortnight. The parietes were tense. There were no enlarged veins upon the surface. There was no evidence of the liver encroaching upon the cavity of the thorax, the chest being resonant on both sides as far down as the lower ribs. The breath and heart-sounds were natural. He had always been moderate as to drink. There was no anasarca anywhere. The urine was very scanty-about six ounces in the twentyfour hours-and loaded with urates; it contained no albumen. There were no symptoms at any time referable to renal disease. He never suffered from jaundice, pain in the right hypochon. drium, or right shoulder. The most distressing symptom appeared to be vomiting, which occurred whenever anything was taken into the stomach-and indeed without. Tongue furred, except at the apex, and tolerably moist; skin cool ; pulse 88, very weak. He had no appetite, as might be expected, and was worn out from want of sleep and from his inability to take nourishment. The bowels had not been opened for five days.

Feb. 16th.-Bowels opened once, scantily; vomiting the same; cannot keep the medicine down.

It is scarcely worth while to go into the treatment from day to day. The object aimed at was to get the bowels to act and to check the vomiting, and for this purpose a number of means were tried, including enemas of warm water and purgatives, and by the mouth seidlitz powders with hydrocyanic acid,- but without avail. He took half a grain of acetate of morphia occasionally, which gave him a few hours' sleep, and refreshed him a little, but the sickness remained the same. The oxalate of cerium was tried, as recently recommended, but with the same unsuccess. The last thing the patient took was chloroform in ten-minim doses, but he sank lower and lower-dying, in fact, by inches, - and finally died on the 10th of March, 1861.

The diagnosis arrived at was obstruction of some part of the intestines, but the exact seat was a disputed point, and upon this hinged the chief interest of the case. The patient was seen by Mr. Lane, one of the honorary surgeons, between whom and $\mathrm{Mr}$. Woodhouse an argument arose: one inclining to the belief that the stricture was in the large intestine, from the history of small and lumpy motions; the other, from tho extreme scantiness of urine, and the absence of all stercoraceous odour in the matters vomited, believing the obstruction to be in the duodenum or thereabouts. The post-mortem examination showed both to be right.

A utopsy.-About three gallons of sanguineous serum wero removed from the cavity of the abdomen. The surface of the peritoneum was universally covered with elevations of a yel. lowish colour, hard to the touch, and in some places semitrans. parent. They varied in size from that of a tare to patches of several inches in area. On the right side, resting against the ribs in the concavity of the diaphragm, was a clot of blood, of a dark colour and rather soft. The omentum was converted into a solid cake, of a hard, fat-coloured character. The trars. verse colon was constricted in its centre by the deposit of this hard, gristly material, but it was pervious to a small extent. On both sides of this constriction was a small amount of semifluid frecs. The large intestine below this to the rectum was contracted and empty. Small intestines healthy and empty. The lesser omentum was thickened by a deposit similar to that found in the larger. Stomach: mucous membrane dark, softened, and stained with bile, but without any deposit in its coats or ulceration. The pylorus was thiclonned and constricted so that it would barely admit a director. The disease appeared. to have commenced on the peritoneal surface, the mucons mem. brane of the two strictured portions not being much affected. A portion of the omentum was placed in St. Thomas's Hospital museum, and Dr. Bristowe, who examined it, stated it to be colloid cancer.

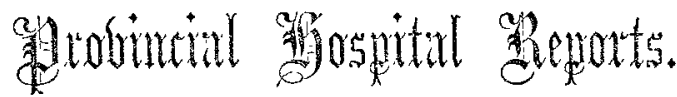

\section{LIVERPOOL NORTHERN HOSPITAL.}

ACUTE RHEUMATISM, WITH PERICARDITIS; TREATMIENT BY OPIUM AND STIMULANTS; RECOVERY.

(Under the care of Dr. WATERS.)

Thomas S- aged seventeen, was admitted April 23rd, 1861. This patient has been the subject of five separate attacks of rheumatic fever during the past eighteen months. On one occasion his chest was affected. About five days before admission he was seized with pain over the hip and arms. Two days subsequently he felt pain at his heart; the latter pain has been increasing. He complains of pain in the knees and hands, over the region of the heart and epigastrium on pressure, and in the left side when he takes a deep breath. The respiration is thoracic; the skin moderately cool. The lungs are resonant, but there is slightly increased extent of dulness in the cardiac region, with increased impulse of the heart; the impulse is felt below and to the left of the left 